\title{
Chanter le poète, et inversement. Evaristo Carriego dans le tango argentin
}

\author{
Pénélope PATRIX (Lisbonne) ${ }^{1}$
}

\section{Summary}

The once-forgotten poet Evaristo Carriego, since rediscovered by Jorge Luis Borges in his eponymous work Evaristo Carriego (1955), is considered today to be one of the leading Argentinian modernist poets, but also, and most importantly, the inventor of a 'poetry of the outskirts' and the creator of one of the major trends in the poetics of contemporary tango, tango canyengue ('rabble' tango).

This article traces the singular trajectory of the poet: first, a singer of the 'low life' of the outskirts of Buenos Aires at the end of the 19th century, of the misfortunes and debauchery of outlaws, prostitutes and the working class; inventor of a 'cantatory' poetry, which draws on popular song and dance of the time, and which we propose to consider as a form of tanguera poetry. Secondly, his influence on the lyricists of contemporary tango leads us to consider him as the precursor of tango canyengue. Finally, he became a founding and guardian figure of contemporary tango, the object of tributes from the greatest composers, and whose name alone has become a poetic and musical trope that invokes a picturesque imagination of the end-of-the-century suburbs of Buenos Aires.

Hence, we are faced with an intermedia corpus, that borrows from the world of popular song to produce a written, erudite and precious poetry, and which in turn becomes one of the main sources of popular song. Carriego's tanguera poetry effectively interlaces word, sound and movement. This paper thus analyses the transmedia trajectory along which the poet sings the tango, and the tango then the poet, in a play of 'inversions' that are indicative of the reciprocal exchanges, transfers and mediations occurring between poetry, song and music, both textual and figurative, from the end of the 19th century to the present day. At the same time, we examine how song, music and dance can 'inhabit' poetry, and poetry 'inhabit' song and music, without constituting, strictly speaking, poetic material or an element of repertoire. It also shows how an authoritative author such as Borges has contributed to shaping the tango tradition by inventing its own genealogy.

\section{Introduction}

L'œuvre du poète argentin Evaristo Carriego (Paraná, 1883 - Buenos Aires, 1912) représente un cas remarquable des relations entre poésie, chanson et musique : ou comment un 
poète mineur de la littérature argentine est devenu une légende littéraire, une figure tutélaire du tango chanté, puis un motif poétique et musical.

Evaristo Carriego est considéré par la critique comme l'un des principaux poètes modernistes argentins, bien qu'il reste, dans la république mondiale des lettres, un écrivain mineur. S'il a joui d'une certaine reconnaissance dans les milieux littéraires de son époque, il a ensuite été relativement oublié, relégué en marge du canon argentin, avant d'être ‘ redécouvert > (ou ‘ révélé >) par Jorge Luis Borges, dans son essai éponyme Evaristo Carriego, initialement publié en 1930, une biographie fictionnelle ${ }^{2}$ qui l'a consacré comme le grand poète du faubourg portègne, et lui a octroyé la place qu'il occupe aujourd'hui dans le champ littéraire argentin, celle de poète mineur de premier plan, si l'on peut dire. C'est en effet le texte de Borges qui a immortalisé le nom de Carriego en littérature, et en a fait une figure littéraire - puisque Carriego est certainement plus connu en tant que sujet de l'essai de Borges, qu'en tant qu'auteur d'une ouvre autonome. Dès lors, et cela en grande partie à travers la lecture qu'en a fait Borges, Carriego s'est mis à incarner le mythe du poète faubourien, inventeur d'une poésie consacrée aux marges, à la ( mauvaise vie >, aux déboires et aventures des petits délinquants et des gens humbles de Buenos Aires du tournant du XX ${ }^{\mathrm{e}}$ siècle (" el compadraje cuchillero y soberbio ", soit " la crapule agricheuse et superbe », Borges [1930] 1974, 106), un univers fantasmé par Borges à travers Carriego, auquel il accorde le difficile rôle de «fondateur » d'une poésie dédiée à ce " monde » disparu (Borges [1930] 1974).

Cette ‘ poésie faubourienne `, habile mélange de raffinement et de débauche, dont Carriego serait l'inventeur - à laquelle il aurait, du moins, donné ses lettres de noblesses - a connu une certaine postérité dans la tradition littéraire argentine, mais aussi, plus notablement, dans la tradition chantée. En effet, toute une veine du tango contemporain (celui qu'on appelle, à partir de Pascual Contursi, Carlos Gardel et d'autres, le tango-chanson, pour le distinguer des formes simples, légères et rudimentaires qui l'ont précédé), dénommée le tango canyengue (tango ‘ canaille ), a repris à son compte cette esthétique, en l'occurrence, pour ce qui nous intéresse ici, dans les paroles des chansons. ${ }^{3}$

Carriego a ainsi été propulsé rétrospectivement en pionnier de la poétique < canaille > du tango, reconnu comme tel par les tangueros eux-mêmes, qui l'ont célébré et lui ont rendu hommage au long du $\mathrm{XX}^{\mathrm{e}}$ siècle. C'est ainsi que le jeune poète oublié est devenu une des figures tutélaires du tango contemporain, dont le nom résonne encore aujourd'hui dans l'imaginaire collectif du tango.

C'est ce parcours transmédial qui constitue le fil rouge de cet article, de la poésie écrite à la musique instrumentale, en passant par l'essai et la chanson; du livre à la tradition chantée et au disque. Il exemplifie les échanges, transferts et médiations réciproques qui s'opèrent entre poésie et chanson au cours du XX ${ }^{\mathrm{e}}$ siècle (‘ inverses `, d'un média l'autre, d'un vers l'autre, comme le suggère le titre). Ou comment le poète chanteur du tango des rues, est devenu le poète des rues chanté par le tango. 


\section{Evaristo Carriego, poète du faubourg}

Evaristo Carriego décède à l'âge de 29 ans, après une courte existence de poète et de journaliste entre 1900 et 1912. Il a grandi à Buenos Aires, dans le quartier de Palermo, qui n’est déjà plus un " terrain vague marécageux en marge de la cité » comme le décrit anachroniquement Borges dans sa biographie fictionnelle du poète (Borges 1930) - sans doute motivé par le besoin d'ancrer le jeune moderniste dans un environnement marginal, périphérique, authentifiant ainsi sa fonction de " poète faubourien " - mais un quartier contrasté, où la partie cossue, celle des jardins élégants et des grandes demeures, jouxte la partie populaire, celle des conventillos et des rives de la rivière du Maldonado, et où quelques enclaves de pauvreté et de criminalité résistent à la planification urbaine, qui sévit alors dans tout le territoire pour transformer la capitale argentine en métropole moderne.

Il fréquente dès son jeune âge les milieux littéraires et intellectuels portègnes ${ }^{4}$ des années 1900, où les écrivains modernistes gravitent autour de Rubén Darío. Il collabore à diverses revues et journaux de son époque, comme Papel y Tinta, le journal anarchiste La Protesta, ou Caras y Caretas, revue satirique à grands tirages spécialisée dans les vignettes de mœurs 5 , dans laquelle il publie ses premiers poèmes à partir de 1906, ce qui lui garantit une audience large et une première reconnaissance de ses pairs.

\section{Une ouvre poétique en deux temps : du modernisme au néo-réalisme}

Il publie son premier recueil de poésie en 1908, Misas herejes (Messes hérétiques), bien reçu par la critique et les milieux littéraires et qui lui apporte une certaine notoriété. C'est le seul livre qu'il publie de son vivant. Un deuxième recueil est publié en 1913 à titre posthume (un an après sa mort), sous le titre La canción del barrio (La chanson du quartier). Le tout est rassemblé peu après dans l'édition complète Poesías de Evaristo Carriego. Misas Herejes, La canción del barrio, Poemas póstumos (Barcelone, 1913). Suivra un recueil de nouvelles, Flor de arrabal (Fleur de faubourg), publié en 1927.

Son œuvre poétique est divisée par la critique, depuis la préface de l'édition de 1913 de son œuvre (Carriego 1913), à la critique plus récente (cf. Borges [1930] 1974, Garcia 2005, Pastormerlo 2018), en deux moments :

- le moment moderniste, qui correspond aux poèmes de jeunesse, d'apprentissage, d'imitation des modèles (Rubén Darío, Almafuerte, Leopoldo Lugones), composé de formes assez conventionnelles (on y trouve une majorité de sonnets espagnols classiques, en hendécasyllabes, et de quatrains conformes au schéma ABBA traditionnel). Cette partie de son œuvre est généralement considérée comme trop figée, un peu empruntée, précieuse, en somme, moins réussie par la critique.

- et le moment que l'on peut appeler ‘ néo-réaliste `, celui où s'impose une voix originale, à la fois personnelle et collective, correspondant à des formes plus inventives (on y trouve de nombreux sonnets où dominent le dodécasyllabe, l'alexandrin et des combinaisons pro- 
sodiques inaccoutumées) et à un langage poétique innovant. C'est celui qui a fait date dans la littérature argentine et qui a consacré Carriego en premier grand poète du faubourg (voir illustration 1).

\begin{tabular}{|l|l|l|}
\hline Titre du recueil & $\begin{array}{l}\text { MISAS HEREJES } \\
\text { (Messes hérétiques) } \\
\mathbf{1 9 0 8}\end{array}$ & $\begin{array}{l}\text { La canción del barrio } \\
\text { (La chanson du quartier) } \\
\mathbf{1 9 1 3}\end{array}$ \\
\hline \multirow{3}{*}{ Sections } & Viejos sermones & La canción del barrio* \\
\cline { 2 - 3 } & Envíos & La costurerita que dió aquel mal paso* \\
\cline { 2 - 3 } & Ofertorios galantes & Íntimas \\
\cline { 2 - 3 } & El alma del suburbio* & Envíos \\
\cline { 2 - 3 } & Ritos en la sombra & Leyendo a Dumas \\
\cline { 2 - 3 } & & Interior \\
\hline
\end{tabular}

Figure 1. Liste des sections composant les deux recueils d'E. Carriego. Les astérisques signalent les sections généralement considérées par la critique comme comprenant les poèmes ‘ faubouriens `.

\section{Le quartier populaire constitué en objet poétique : bords et débords}

Mais il serait réducteur de limiter l'émergence du "poète des faubourgs " à cette seconde partie de l'œuvre de Carriego - dès Misas herejes, les poèmes mettent en place cette poésie des bords, alliance contradictoire du raffinement et du prosaïsme qui fait toute la force de sa poésie faubourienne. Dans toute son œuvre poétique, Carriego se fait le chantre du quartier populaire, de la rue, des ' petites gens `, des marginaux et des laissés pour compte, pour reprendre les formules de Borges (Borges [1930] 1974).

Il constitue le quartier populaire en objet poétique : le ‘ faubourg >, sa galerie de personnages types et son univers prosaïque, deviennent sujet de sa poésie, ce en quoi il effectue un déplacement par rapport aux sujets poétiques traditionnellement valorisés, en prenant pour cadre, non seulement la ville moderne, mais ses bords. Mieux, ils en deviennent la matière, à travers des effets de citation, d'emprunts, d'imitation formelle, engendrant de ce fait une série de motifs, de scénarios et d'images inédits. Le lunfardo (l'argot local) ${ }^{6}$ et les formes du langage oral viennent s'entrelacer aux raffinements du langage érudit, des références savantes se mêlent à des références à la culture populaire, les formes et schémas classiques de la poésie rencontrent la dissonance ou l'imperfection du vers, l'effronterie > canaille > se heurte à la mélancolie des déshérités, dans un entrechoc tout à fait nouveau.

Carriego réussit en quelque sorte à produire une synthèse originale entre le modernisme et la poésie réaliste, en argot (appelée poésie lunfarda ou lunfardesca en Argentine) ; avec lui, l'argot et le faubourg prennent leurs quartiers, non pas en littérature, puisqu'il se réapproprie une tradition de poésie argotique issue de la littérature populaire, mais dans la littérature savante. C'est cette sophistication qui fait dire à Borges (et à quelques-uns de ses 
contemporains - voir la préface à l'édition complète de ses poèmes en 1913) que Carriego est le premier s grand s poète du faubourg. Autrement dit, Carriego invente une lyrique du faubourg moderne (de même que Baudelaire, qui n'est sans doute pas le premier poète de la rue, a inventé une lyrique de la ville moderne).

Tout un imaginaire pittoresque des faubourgs de Buenos Aires, de ses bords et débords, associé aux milieux de la pègre, des bas-fonds et de la ‘ mauvaise vie `, qui envahit à cette époque les journaux, les enquêtes criminelles, les règlementations politico-sociales, le théâtre et la littérature, en Argentine et dans le monde (Caimari 2013, Kalifa 2013), est ainsi convertie en matériau poétique.

Dans cette poésie faubourienne, l'esprit gaillard, allègre, frondeur et indiscipliné de la ( crapule ', caractéristique de la poésie lunfarda, s'allie à l'expression de la douleur, de la misère, de la solitude, mais aussi de la violence et des débordements des ‘ déviants >, produisant une forme de tragique faubourien (que Borges appelle quant à lui une "épique du faubourg", "épica del suburbio » - cf. Borges [1930] 1974). Il chante les joies obscènes mais aussi les désillusions des voyous, des filles de joie, des blanchisseuses et des vagabonds (les compadritos et les chinas, dans leur version portègne).

L'exemple qui suit est justement extrait du poème "A Colombina en carnaval ", issu de la section «Ofertorios Galantes » des Misas herejes:

Modista, pobre tendera,

o esclava del obrador:

vestida de primavera,

ya rendirás al hortera,

tenorio de mostrador.

Flor que aroma el delincuente

búcaro del Cafetín,

loca máscara insolente

que aguarda lista, impaciente,

su gallardo bailarín.

Ebrio de amor y de vino, sensual donaire guarango

lucirá tu cuerpo fino,

esta noche en el Casino

cuando te entusiasme el tango.

Muchacha conventillera

que, en apuros maternales,

pasaste la noche entera
Modiste, pauvre petite boutiquière,

ou esclave de l'atelier :

dans ta tenue printanière,

tu vaincras le plouc,

don juan de comptoir.

Fleur qui embaume le délinquant

cruche du Troquet,

masque insolent et fou

qui attend, prête, impatiente,

son gaillard danseur.

Ivre d'amour et de vin,

dans sa sensuelle grâce de crapule,

il exhibera ton corps fin,

cette nuit au Casino

quand le tango t'exaltera.

Jeune fille des conventillos

qui, avec un soin maternel

a passeé la nuit entière 
arreglando esa pollera, honra y prez de los percales,

ya, despertando las ganas

de otras de la vecindad,

irás con tus dos hermanas,

Terpsicores suburbanas,

a un baile de sociedad... à arranger ce cotillon,

honneur et gloire des percales,

bientôt, suscitant l'envie

des filles du voisinage,

tu iras avec tes deux sœurs,

Terpsichores faubouriennes,

à un bal de société...

(Carriego 1913, 72 ; trad. P.P.)

Sans entrer dans une analyse formelle trop détaillée, on se contentera ici de relever la prosodie octosyllabique régulière de ce poème, composé de strophes de cinq syllabes (les quintillas), de vers disposés librement - dans la troisième strophe, tous les vers riment entre eux, ce qui transgresse la règle classique, issue de l'arte menor, pour se rapprocher d'une forme plus libre, non conventionnelle.

Le poème élabore un imaginaire des faubourgs, à travers une topologie faubourienne, qui a pour marqueurs l'atelier, le troquet, le Casino, le conventillo, le ‘ voisinage `, le bal public ; et une série de personnages, avec leurs caractéristiques bien marquées, le voyou, ou ‘ délinquant , séducteur, buveur, "gaillard danseur ", la grisette, ici sous les traits de la modiste, partenaire du voyou, qui revêt, avec l'allusion à Colombine allant au carnaval, le ‘ masque insolent > de la commedia dell'arte - insolent par la transgression poétique même qu'il suscite.

Ces attributs entrecroisent des références érudites, comme Terpsichore, muse de la danse dans la mythologie grecque. Cela donne lieu à des figures insolites mais fulgurantes, qu'on peut qualifier d'oxymoriques (des oxymores culturels, en quelque sorte), dans la fusion paradoxale des codes qu'elles créent, comme le "don juan de comptoir " (" tenorio de mostrador »), ou la "fleur qui embaume le délinquant " ( Flor que aroma el delincuente »), où l'on reconnaît une forme paradigmatique de la " fleur de bitume » universalisée par la littérature et la chanson ‘ canailles > de l'époque.

Les occurrences de lunfardo, de langage familier et cru, sont associées à des tournures sophistiquées, des termes précieux, des symboles et des allusions, dans un usage ludique et poétique qui contribue à produire ces effets de sublime gouaille.

Il faut souligner que le texte fait référence à la culture populaire urbaine de son époque, et plus particulièrement ici, au tango. Le tango est même un élément central de ce poème, c'est par lui que s'unissent les deux figures masculine et féminine. C'est un tango fortement sexualisé, un tango ivre, associé à l'exaltation procurée par l'amour et le vin. Des caractéristiques typiques de la débauche telle qu'elle est décriée à l'époque (l'ivrognerie, l'obscénité des danses populaires, la perversion des jeunes ouvrières, la dépravation des délinquants) sont ici transformées en valeurs d'hédonisme et de sensualité. Là aussi, cela donne lieu à une formule originale, paradoxale, la "sensuelle grâce de crapule " (" sensual donaire guarango »), qui, une fois de plus, a pour effet de sublimer la figure du voyou danseur de tango. La grisette et 
le voyou des faubourgs deviennent ainsi des figure poétiques, exaltées par cette poésie, qui prend à contrepied (on note ici l'application poétique d'une figure de la danse de couple) les conceptions stéréotypées de la délinquance, en retourne les stigmates, pour construire un nouveau stéréotype littéraire, celui de la superbe faubourienne.

\section{Une poésie tanguera avant la lettre}

Et en effet, l'une des spécificités de la poésie de Carriego est qu'elle est imprégnée de références à la musique, à la chanson et à la danse. Le poème « Lâme du faubourg » ("El alma del suburbio ") en offre un exemple éclatant :

El gringo musicante ya desafina en la suave habanera provocadora, cuando se anuncia a voces, desde la esquina "el boletín - famoso - de última hora".

Entre la algarabía del conventillo, esquivando empujones pasa ligero, pues trae noticias, uno que otro chiquillo divulgando las nuevas del pregonero.

\section{[...]}

En la calle, la buena gente derrocha sus guarangos decires más lisonjeros, porque al compás de un tango, que es "La Morocha"

lucen ágiles cortes dos orilleros.

La tísica de enfrente, que salió al ruido, tiene toda la dulce melancolía de aquel verso olvidado, pero querido, que un payador galante le cantó un día.

La mujer del obrero, sucia y cansada, remendando la ropa de su muchacho, piensa, como otras veces, desconsolada, que tal vez el marido vendrá borracho.
Le gringo musicien fait déjà dissoner la habanera suave et provocante, quand on annonce à pleine voix, au coin de la rue "la gazette - fameuse - du soir ».

Dans le joyeux vacarme du conventillo esquivant les coups, se glisse rapidement, pour apporter les nouvelles, un des gamins qui colporte les annonces du crieur public.

\section{[...]}

Dans la rue, les braves gens prodiguent des grossièretés des plus flatteuses, car au rythme d'un tango, qui est "La Morocha " deux faubouriens exécutent d'agiles cortes.

La phtisique d'en face, qui est sortie à ces bruits, éprouve toute la douce mélancolie de ce vers oublié, bien qu'aimé, qu'un galant payador lui a chanté un jour.

La femme de l'ouvrier, sale et fatiguée, raccommodant les vêtements de son gars, pense, comme bien des fois, affligée, que sans doute son mari rentrera saoul.

(Carriego 1913, 81-82 ; trad. P.P.) 
Ce poème élabore toute une écologie sonore du quartier, composée de bruits, de voix ، dissonantes ', de cris, de rumeurs, des éclats d'une bagarre, d'injures, de musique et de chant (Monteleone 2006), créant un effet global de cacophonie urbaine, un ‘ joyeux vacarme `. Il se fait réceptacle - mieux, caisse de résonnance - de la vie du quartier populaire de Buenos Aires.

Parmi les genres cités, on trouve la chanson de payador (le chanteur ambulant des campagnes et des faubourgs, qui improvise des vers en s'accompagnant à la guitare), la habanera, et le tango, avec une référence à " La Morocha ", une chanson à succès de 1905, composée par Ángel Villoldo et Enrique Saborido. À travers ces références à la musique et à la danse, le poème tente de saisir l'، âme > du faubourg. Chacune est chargée d'une émotion propre, la habanera, "suave et provocante ", le tango, ses figures " agiles " et ses paroles " grossières ", qui suscite le souvenir de chansons de payadores et la " douce mélancolie " d'une voisine. Sont ainsi entremêlés, selon un art du contrepoint qu'on a déjà noté par ailleurs, des affects contraires, qui créent toute la complexité et la subtilité de ces mélodrames de faubourgs.

Le tango en particulier occupe une place de choix dans la poétique de Carriego. Il est constitué en indice (au sens sémiologique de signe indiciaire) du monde ‘ canaille >, suffisant à lui seul à convoquer un imaginaire exubérant de la pègre, de la " mauvaise vie " (mala vida) des faubourgs portègnes, qui sont, comme on l'a vu précédemment, grâce à un usage poétique du contrepoint, sublimés. Autrement dit, Carriego fait du tango - un tango ‘ canaille , sensuel, agile et égrillard - un topos du monde de la rue.

Mais le tango, et plus généralement la musique et la danse, deviennent aussi, chez Carriego, des motifs poétiques, au sens le plus concret du terme : le poème réinvestit des codes, des rythmes, des figures, des paroles, des schémas narratifs propres au tango et plus généralement à la musique populaire du Rio de la Plata. C'est pourquoi je propose de parler de poésie tanguera: le poème fait résonner, dans sa matière même, paroles, sons et motifs chorégraphiques - il fait chanter et danser la poésie. Ce faisant, le poème ‘ chante > le tango. Roberto Giusti écrit à ce titre dès 1911 que, comme le résume Pastormerlo $(2018,8)$, « le rythme des poèmes de Carriego semblait en quête des pas du tango (" el ritmo de los poemas de Carriego parecía estar buscando el andar del tango ») :

Il me semble même que l'âme musicale du faubourg est entrée dans les Messes hérétiques. Le vers que Carriego utilise avec prédilection, le dodécasyllabique accentué à la sixième [syllabe], dont on dirait qu'il se " brise » en une cuerpeada [figure du tango], semble conserver le rythme plein de souffle du tango que les organillos [petits orgues de rue] des faubourgs ont fait couler dans les oreilles du poète. ${ }^{8}$

Certains vers ou poèmes sonnent comme des réminiscences de paroles de tango, en empruntant les formules et les figures. Ainsi, "La Morocha », ce tango cité dans «El Alma del Suburbio ", comprend par exemple les vers "Soy la gentil compañera / del noble gaucho porteño ", vers dont les termes sont redistribués dans le poème. La chanson vient ainsi s'insérer dans le poème, sa mélodie et ses paroles opérant comme un sous-texte. Une inter- 
textualité forte entre le poème et la chanson populaire est créée, sans pour autant que soient brouillées les limites entre le poème et le chant, puisque, comme on l'a vu, le poème investit ces éléments dans une écriture bien spécifique, avec sa prosodie et ses référents propres. Borges parle à juste titre, pour souligner le geste de déplacement, effectué par Carriego, des " épanchements incongrus de ce poète spécialiste du faubourg ». Incongrus, car il ne s'agit pas d'imiter simplement les formes du tango chanté, mais de les transposer (comme un compositeur arrange des airs populaires, pour produire des œuvres originales, mais qui charrient comme des souvenirs de mélodies, de refrains).

Certains ont vu dans ces poèmes ‘ cantatoires ', rétrospectivement, les prémisses du tango-chanson à venir, écrit par des poètes comme Pascual Contursi ou Celedonio Flores, ceux-là même qui allaient, à partir des années 1910, donner ses ‘ lettres de noblesse ` au tango chanté. Comme si les poèmes de Carriego renfermaient déjà l'esprit du tango ( savant >, dit " poétique », constituaient une poésie tanguera avant la lettre. Ainsi, pour Sergio Pastormerlo $(2018,8)$, Carriego " écrit des poèmes de tango ». D'après Conrado de Lucia, Leonardo Castellani aurait qualifié cette œuvre de "bastante tangusa " ( assez tangusa », un néologisme), tandis que lui-même parle, de façon encore plus directe et anachronique (et donc forcément métaphorique), des « tangos de Carriego » (Lucia 2001).

\section{Evaristo Carriego, précurseur du tango canyengue}

La poésie faubourienne inventée par Carriego a certainement eu une influence décisive sur les paroliers de tango des années 1910 à 1930, dits de la « Vieille Garde `. C'est principalement dans la chanson tango qu'il a trouvé sa postérité. ${ }^{9}$ C'est pourquoi il est considéré à maints égards comme le précurseur de la poétique du tango-chanson moderne, et plus particulièrement de l'esthétique ‘ canaille > (canyengue) du tango du XX $\mathrm{XX}^{\mathrm{e}}$ siècle, ce tango qui célèbre les marginaux des faubourgs et la vie des faubourgs.

Par exemple, son usage figuré (métonymique) du mot ‘ percale `, pour désigner la jeune fille des faubourgs (vêtue de percale, soit d'extraction populaire, par opposition aux femmes vêtues de soie), dont on trouve une occurrence dès le poème "A Colombina en carnaval " cité plus haut, deviendra un topos de la poésie tanguera.

De nombreux éléments de la poétique de Carriego, telle que je viens de l'examiner, seront transposés dans le répertoire chanté : les figures archétypales qu'il élabore, notamment le duo du voyou et de la fille, devenu emblématique du tango canyengue, l'association du lunfardo à une écriture sophistiquée, maniérée même, qui revendique une forme d'élégance décadente, ses figures oxymoriques, devenues des topö̈, la tension créée entre l'allégresse ‘ canaille >, le mélodramatisme et la mélancolie, les références métapoétiques au chant et à la danse, et plus généralement, l'élaboration poétique d'un système de représentation de la ( mauvaise vie . portègne des débuts du XX $\mathrm{XX}^{\mathrm{e}}$ siècle. Guillermo García a bien montré comment, à la suite de Carriego, le ‘ faubourien > comme esthétique est entré dans le répertoire du tango-chanson. Il a analysé les effets d'écho, de reprise et d'allusions aux poèmes de Carriego chez les grands 
paroliers du tango (García 2005). Son style poétique a contribué à former le < langage > du tango, et a eu une grande influence sur des paroliers et des poètes comme José González Castillo, Celedonio Flores pour les années 1920, Enrique Cadícamo, par exemple dans «El último guapo " ("Le dernier marlou »), Eduardo Calvo dans "Arrabalero » (" Faubourien »), Enrique Santos Discépolo dans "El malevaje " ( La pègre »), une lamentation de voyou, pour les années 1930, Homero Manzi dans les années 1940, qu'Horacio Ferrer considère comme son plus grand héritier (Ferrer 1977, cité dans Lucia 2001). Ferrer considère d'ailleurs lui aussi Carriego, à la suite de Borges, comme "le premier grand poète du faubourg portègne " ("el primer gran poeta del suburbio porteño »), et les paroles de Manzi comme des " oraisons à la manière de Carriego " (" oraciones al modo de Carriego »).

En somme, nous sommes face à une ouvre intermédiale, qui s'est inspirée de la chanson et de la danse populaires de son époque, pour produire une poésie tanguera, et a ensuite été réappropriée à son tour dans l'écriture du tango chanté, pour produire un tango poétique. Dans le cas qui nous occupe ici, il semblerait que si Carriego a eu un tel impact sur la poétique du tango, c'est que sa poésie contenait déjà en germe quelque chose de tanguero, une tension vers le chant, la voix, la musique et la danse, du fait même qu'elle s'en était inspirée. Il ne suffit donc pas de repérer les situations où des poèmes sont mis en chanson, pour montrer que la chanson trouve sa source dans la poésie ; il faut également identifier celles, comme ici, où la chanson est mise en poème, où la poésie trouve sa source et sa matière dans la chanson, perpétuant en cela, à l'orée du XXe siècle, la longue tradition, qui remonte au Moyen Âge, de réappropriation des chansons populaires dans la poésie écrite.

Les poèmes de Carriego ont sans doute été lus par Rosendo Mendizabal (mort 1913), Ángel Villoldo (mort en 1919), et d'autres de ses contemporains, dans les revues et journaux de l'époque (tout comme d'autres poèmes lunfardos avant lui, et d'autres poètes publiés dans la presse - il ne s'agit surtout pas d'ériger Carriego en source poétique unique du tango-chanson). C'est ce que défendent des spécialistes comme Horacio Ferrer et Guillermo Garcia. Mais au-delà de cette influence directe sur les premiers paroliers du tango, c'est certainement de façon indirecte et progressive que sa poétique faubourienne est entrée dans le répertoire tanguero. Plus que son œuvre, c'est son style poétique qui a été incorporé dans la chanson tanguera, sans que les paroliers l'aient nécessairement lu ou se soient référé à lui, et qui est entré dans la tradition chantée.

Carriego a ainsi produit un canon pour le tango contemporain - sans avoir lui-même, c'est toute l'ironie de cette histoire, jamais écrit un seul tango ${ }^{10}$; et sans que, autre ironie de l'histoire, ses textes aient eux-mêmes été mis en musique et interprétés par les chanteurs et chanteuses de tango de son époque. Comme l'avance Sergio Pastormerlo « [E. Carriego] s'est intégré à l'histoire du tango, selon le destin ironique des précurseurs, passé dans l'oubli et fondu dans la tradition qu'il avait initiée : ses poèmes n'ont guère donné de paroles de tango $»^{11}$. 


\section{Evaristo Carriego chanté dans le tango : tangentes}

Cela nous amène au troisième et dernier temps de ce parcours transmédial, soit quand (et comment) le tango a chanté le poète.

Evaristo Carriego n'a pas écrit de tangos à proprement parler - de paroles pour le tango, car il faut bien distinguer l'écriture ‘ cantatoire , du poète, de l'exercice qui consiste à composer un texte destiné à être chanté. Par ailleurs, ses textes n'ont pas été interprétés par les chanteurs et chanteuses de tango de son époque - confirmant la relative étanchéité qui se maintient, du point de vue pragmatique, entre une poésie écrite, une écriture, et une poésie vocale, écrite pour la voix. En effet, Carriego n’a pas, paradoxalement, été mis en chanson, même postérieurement, au cours du $\mathrm{XX}^{\mathrm{e}}$ siècle. Ses poèmes ne sont pas devenus un matériau du chansonnier tanguero contemporain. De ce fait, ses poèmes sont absents, en tant que matériau textuel, ou corpus, du répertoire tanguero. ${ }^{12}$ Le grand poète du faubourg, le grand inspirateur du tango ‘ canaille > moderne, a donc eu, proportionnellement, peu de visibilité (ou d'audibilité) dans le répertoire tanguero - incorporé, puis oublié, bien moins chanté qu'on ne pourrait s'y attendre. Son influence sur la chanson a été indirecte, fruit d'une médiation, consistant de la part des paroliers du tango à transposer son style poétique dans la composition de paroles. D'où la distinction, sur laquelle j'insiste un peu, entre ses poèmes (un corpus de textes) et sa poétique (un style) - c'est sa poétique qui a été réinvestie par le tango canyengue, plutôt que ses poèmes. On se trouve donc face à un phénomène incongru, comme je l'ai souligné, où Carriego est reconnu comme une figure tutélaire du tango canyengue, dont la poétique a été assimilée dans la tradition tanguera, sans que ses textes soient entrés, en tant que tels, dans le répertoire du tango - un modèle assimilé au point qu'il hante le répertoire, sans en faire, concrètement, partie, sorte de présence-absence, de sous-texte du tango chanté.

Et en effet, c'est selon une autre voie, que le tango contemporain a ' chanté le poète ) : en célébrant son nom dans le tango, un simple nom qui suffit à lui seul à convoquer tout un imaginaire poético-musical de la bohème portègne, de la rue et du tango ‘ canaille > - un nom devenu lui-même motifpoétique, en somme. Dès $1926^{13}$, le poète et parolier Homero Manzi le consigne dans la chanson "Viejo ciego » ("Vieil Aveugle») :

pareces un verso

del loco Carriego

pareces el alma

del mismo violin ${ }^{14}$ tu sembles être un vers

de ce fou de Carriego

tu sembles être l'âme

du violon lui-même

Le texte s'adresse au "vieil aveugle ", musicien itinérant ${ }^{15}$, posé en incarnation du tango bohème. Par un jeu de contaminations et d'inversions du système symbolique conventionnel, la figure de l'aveugle incarne l'univers poétique de Carriego, la figure même du poète (le "fou "), « l'âme » du violon (on dit généralement que le violon contient l'âme du poète). C'est évidemment du poème «Viejo ciego » lui-même qu'il s'agit in fine, inscrit de la sorte 
dans la filiation de Carriego, ce poète dont le tango perpétue la mémoire en le thématisant au sein même du poème. L'aveugle est une figure récurrente de la poésie de Carriego - qu'on pense par exemple au poème " Has vuelto » (« Tu es revenu »), compris dans « La canción del barrio " du recueil posthume du même titre, où l'on retrouve le même jeu de contamination entre la figure de l'aveugle, l'instrument de musique (l'organillo, auquel s'adresse ici le poème, inversement), et tout un imaginaire symbolique que leur présence même suscite. ${ }^{16}$ La chanson de Manzi convoque comme en palimpseste la figure type du Vieil aveugle créée par Carriego, et du même geste, la mémoire du poète. Manzi réitère ce jeu de citations, et d'échos réciproques entre poésie et chanson, dans "El último organito ", où le nom de Carriego refait surface : "El ciego inconsolable del verso de Carriego ». Ici la référence au poème de Carriego est explicite. Et encore une fois, c'est le nom même de Carriego, inscrit dans le texte de la chanson, qui véhicule la mémoire de sa poésie.

Si le tango lui-même a perpétué son nom, que les interprètes successifs des chansons de Manzi vont successivement faire résonner et rendre au souvenir des auditeurs de tango, c'est bien l'hommage qui lui a été rendu par Borges en 1930 qui constitue Carriego, ce poète que la littérature a, entre-temps, plus ou moins oublié, en légende littéraire, en poète pionnier, et en figure fondatrice du tango canyengue, affectant son nom d'une prodigieuse charge symbolique. C'est le poète tel que l'a célébré Borges, autrement dit, le Carriego de Borges, qui a été consacré comme le père du tango faubourien dans la mémoire collective. Comme l'affirme Sergio Pastormerlo, "En publiant son Evaristo Carriego en 1930, Borges a ratifié et amplifié le mythe : dorénavant, le personnage borgésien a quasiment prévalu sur le Carriego historique $»^{17}$.

Dès lors, le "Carriego » ravivé par Manzi et honoré par Borges, s'impose en figure tutélaire du tango canyengue en faisant l'objet d'hommages et de dédicaces de la part des compositeurs, paroliers et interprètes du tango contemporain. C'est ainsi que celui qui avait célébré le tango des faubourgs, devient lui-même, par contamination, le " poète $d u$ faubourg " (avec toute l'ambiguïté que véhicule l'article partitif, poeta del arrabal), et est célébré comme tel dans le tango des grands orchestres et des artistes consacrés du XXe siècle.

Parmi ces hommages musicaux, l'on peut citer la chanson "Carriego " (paroles de Julio Jorge Nelson, musique de Roberto Nievas Blanco), enregistrée par Roberto Arrieta avec l'Orquesta Miguel Caló en 1948 (chez Buenos Aires Odeon). La chanson rend hommage au poète, à son "émotion de faubourg " (" emoción de suburbio ") ${ }^{18}$, mais effectue aussi, par la même occasion, une formidable interprétation critique de son œuvre et de sa réception. Mais l'hommage le plus célèbre, celui qui a sans doute le plus contribué à perpétuer le nom de Carriego dans le tango, est donné, non par une chanson, mais par un tango instrumental : "A Evaristo Carriego ", d'Eduardo Rovira, interprété et enregistré par Rovira lui-même en trio en 1966 (Orchard music) ${ }^{19}$, puis par de nombreux orchestres, comme le fameux Orquesta Osvaldo Pugliese, qui l'enregistre successivement en 1969, en 1985, et en 1989 avec Astor Piazzola ${ }^{20}$. Dans ce cas-limite, qui vient clore notre parcours, le poète est chanté sans paroles. C'est sous forme de réminiscences, de traces mémorielles, que son œuvre poé- 
tique est ravivée, opérant tel un sous-texte de la musique mais aussi par la charge signifiante que son nom, simplement cité, accorde à la musique qui le nomme.

\section{Notes}

1 Pénélope Patrix est chercheuse au Centro de Estudos Comparatistas de la Faculté de Lettres de l'Université de Lisbonne. L'écriture de cet article a été financée par des fonds nationaux portugais à travers la FCT - Fundação para a Ciência e a Tecnologia, I.P., dans le cadre du projet UIDB/00509/2020. L'auteure bénéficie d'une bourse postdoctorale du Centro de Estudos Comparatistas (UID/ELT/00509/2013 [THELEME - RG-509-1999]).

2 Voir le court prologue de Borges dans ses Euvres complètes sur ce livre " moins documentaire qu'imaginaire " (" menos documental que imaginativo »; Borges 1974, 101).

3 Bien que l'esthétique ‘ canaille > du tango ne se limite évidemment pas aux paroles, mais se manifeste également dans la musique, la danse, les costumes, les scénographies, l'iconographie (cf. Patrix 2014).

4 (Portègne `, traduction de porteño, gentilé de Buenos Aires. « Portègne > signifie : [originaire] de Buenos Aires.

5 Sur cette revue, premier journal illustré de masse en Argentine, cf. Garabedian/Szir/Lida 2009.

6 Formalisé au même moment par la police et la criminologie comme le langage secret des milieux du crime, propre aux compadrito, le voyou typique de Buenos Aires, et dont les listes et dictionnaires constitués par les policiers < de terrain > sont appropriés par les écrivains de la littérature populaire et de la poésie lunfarda (soit ' poésie en argot ', correspondant à ce qu'en France on appelle la ( poésie de pègre `). Le lunfardo devient également le marqueur d'un langage local, vernaculaire, typiquement portègne et citadin en littérature, dans le prolongement de la littérature gauchesca, qui trouve dans le gaucho l'équivalent rural du compadrito, au moment où l'Argentine, comme bien d'autres pays du monde, constitue sa littérature nationale (Conde 2011).

7 Ces analyses de la poésie de Carriego, et plus généralement de la ' poétique canaille , dans la culture de l'Argentine fin-de-siècle, ont été en partie formulées dans ma thèse de doctorat (Patrix 2014).

8 « Hasta se me antoja que ha entrado en Misas herejes el alma musical del suburbio. El verso que Carriego usa con predilección, el dodecasílabo acentuado en la sexta, que diríase quebrarse en una cuerpeada, parece conservar el ritmo fuelle del tango que los organillos arrabaleros molieron en los oídos del poeta ». (Roberto Giusti, Nuestros poetas jóvenes, 1911, cité dans : Pastormerlo 2018, 8 ; trad. P.P.).

9 Carriego a également eu une influence sur la poésie réaliste argentine, la poesia dite lunfarda, ou arrabalera, ( faubourienne , comme celle de Felipe "Yacaré » Fernández, de Carlos de la Púa, d'Iván Dize. Mais c'est sa filiation dans la chanson que je voudrais suivre ici. Voir sur ce point Ferriol (2017).

10 À ma connaissance, du moins, et selon la critique citée. 
11 « [EC] se integró a la historia del tango, según el destino irónico de los precursores, pasando al olvido y fundiéndose en la tradición que iniciaba: sus poemas apenas dieron alguna letra de tango. " (Pastormerlo 2018, 8 ; trad. P.P.). Cette dernière affirmation serait évidemment à prendre avec précaution, comme je tente de le faire ici. Je pense que Pastormerlo se réfère strictement au fait que les poèmes de Carriego n’ont pas été chantés (du moins, pas enregistrés) par ses contemporains (du moins, les plus célèbres). Ici encore, cela reste une hypothèse.

12 Concernant les potentielles mises en musique et en voix des poèmes de Carriego, la seule occurrence que j'aie trouvée de chanson dont le texte soit attribué à Carriego se trouve sur le site internet Todotango sous le titre "La muerte del pibe » ("La mort du gamin »), sur une musique de Emilio Sola et de Raymundo Cabarcos, enregistrée par Domingo Conte en 1935 (http:/www.todotango.com/musica/tema/1979/La-muerte-del-pibe/). Mais je n’ai trouvé aucun enregistrement de cette chanson, et surtout, le poème en question n'est pas inclus dans les œuvres complètes du poète. Il s'agit donc sans doute d'une attribution erronée.

13 Manzi aurait écrit le texte en 1925, soit à l'âge de 14 ans, sous le titre "El alma que canta ", titre qui évoque «El alma del suburbio » de Carriego, cité plus haut. Elle est donnée pour la première fois au théâtre, par Roberto Fugazot, en 1926. La même année, paraît le film muet La costurerita que dio aquel mal paso de José Agustín Ferreyra, dont le titre renvoie au poème de Carriego du même nom, et qui s'ouvre sur une réécriture du poème. On voit donc qu'avant Borges, Carriego a déjà été incorporé dans la culture populaire.

14 «Viejo ciego », paroles de Homero Manzi, musique de Sebastián Piana et Cátulo Castillo (1926). Le texte intégral peut être consulté sur le site Todotango (http://www.todotango.com/musica/ tema/371/Viejo-ciego/), de même que l'interprétation de Roberto Goyeneche dans un enregistrement de 1990.

15 Comme l'indique le premier couplet :

«Con un lazarillo llegás por las noches

trayendo las quejas del viejo violín,

$y$ en medio del humo

parece un fantoche

tu rara silueta

de flaco rocín.

Puntual parroquiano tan viejo y tan ciego,

al ir destrenzando tu eterna canción,

ponés en las almas

recuerdos ańejos

y un poco de pena mezclás al alcohol. »

16 Voir la première strophe de "Has vuelto » (Carriego 1913, 183) :

«Has vuelto, organillo. En la acera

hay risas. Has vuelto llorón y cansado

como antes.

El ciego te espera

las más de las noches sentado 
a la puerta. Calla y escucha. Borrosas

memorias de cosas lejanas

evoca en silencio, de cosas

de cuando sus ojos tenían mañanas

de cuando era joven... la novia... ¡quien sabe!

Alegrías, penas,

vividas en horas distantes. ¡Qué suave

se le pone el rostro cada vez que suenas

algún aire antiguo ¡Recuerda y suspira!

$[\ldots] »$

17 «Al publicar su Evaristo Carriego en 1930, Borges ratificó y amplificó el mito: desde entonces el personaje borgeano casi prevaleció sobre el Carriego histórico. » (Pastormerlo 2018, 1 ; trad. P.P.).

18 Les paroles peuvent être consultées en ligne sur le site Hermanotango.com (http://www.hermanotango.com.ar/LetrasTangos/CARRIEGO.htm).

19 L'enregistrement peut être écouté en ligne sur YouTube (https:/www.youtube.com/ watch?v=665X8YCF-Hc).

20 L'enregistrement en direct effectué au Teatro Colón en 1985 peut être écouté sur YouTube (https://www.youtube.com/watch?v=imlf-BR7J9w), ainsi que celui qu'il a effectué en studio à Amsterdam avec Astor Piazzola en 1989 (https://www.youtube.com/watch?v=ZEr-G9ocA3c).

\section{Bibliographie}

Borges, Jorge Luis : «Evaristo Carriego [1930] ». In : Borges, Jorge Luis : Obras completas (19231972). Buenos Aires : Emecé Editores, 1974, 97-172.

Borges, Jorge Luis : Evaristo Carriego. Trad. Françoise Rosset. Paris : Seuil, 1969.

Caimari, Lila : «Police, tango et argot : culture policière et culture populaire à Buenos Aires au XXe siècle ». In : Histoire, économie \& société 4 (2013), 41-48.

Carriego, Evaristo : Poesías. Misas Herejes, La Canción del Barrio, Poemas postúmos. Barcelona : Auber y Pla, 1913.

Conde, Oscar : Lunfardo. Un estudio sobre el habla popular de los Argentinos. Buenos Aires : Taurus, 2011 [format kindle].

Ferrer, Horacio : El libro del tango. Buenos Aires : Galerna, 1977.

Ferriol, Ezequiel : « Barrio, academia y realismo en « Amablemente `, de Iván Diez ». In : Fua Púppulo, Violaine / Curcio, Jorge (éds) : Primeras jornadas de lenguaje, literatura y tango : cruces entre la lungüística, la crítica literaria y el psicoanálisis. Buenos Aires : La Docta Ignorancia, 2017, 248-259.

Garabedian, Marcelo H. / Szir, Sandra M. / Lida, Miranda : Prensa argentina, siglo XIX: imágenes, textos y contextos. Buenos Aires: Teseo, 2009.

García, Guillermo: "El arrabal como hecho estético: la poesía popular de Evaristo Carriego y su aporte a la identidad rioplatense ». In : Espéculo. Revista de estudios literarios 30 (2005), 
https://webs.ucm.es/info/especulo/numero30/carriego.html (consultation 15.06.2020).

Gobello, José : Nuevo diccionario lunfardo. Buenos Aires : Corregidor, 2003.

Kalifa, Dominique : Les bas-fonds. Histoire d'un imaginaire. Paris : Seuil, 2013.

Lucia, Conrado de : «Los tangos de Evaristo Carriego ». In : Revista Bondiguía 4 (2001), republié sur le site Terapia Tanguera : http://www.terapiatanguera.com.ar/Notas\%20y\%20articulos/carriego. htm (consultation 15.06.2020).

Monteleone, Jorge : "La invención de la ciudad. Evaristo Carriego y Baldomero Fernández Moreno ». In : Rubione, Alfredo (éd.) : La crisis de las formas. Historia crítica de la literatura argentina. Vol. 5. Buenos Aires : Emecé, 2006, 205-235.

Pastormerlo, Sergio : "Evaristo Carriego. Cantar y contar las voces del barrio ». In : García Cedro, Gabriela / Viñas, David (éds) : Literatura argentina siglo XX. Buenos Aires : Paradiso (à paraître). Épreuve du texte accessible sur la page personnelle de l'auteur : https://www.academia.edu/33902607/Evaristo_Carriego_Cantar_y_contar_las_voces_del_barrio (consultation 15.06.2020).

Patrix, Pénélope : Imaginaire des bas-fonds et poétique ‘ canaille > dans la chanson urbaine. Le fado et le tango, des marges au patrimoine immatériel. Thèse de doctorat. Université Paris Diderot 2014.

\section{Discographie}

Arrieta, Roberto / Orquesta Miguel Caló : "Carriego ». In: Caló, Miguel y su orquesta típica : Sus éxitos con Roberto Arrieta. EMI/DBN 724349997127, 1999 (CD).

Melingo, Daniel : Corazón \& Huezo. World Village WVF479064, 2011 (CD).

Melingo, Daniel : Linyera. World Village WVF 479089, 2014 (CD).

Pugliese, Osvaldo y su orquesta : "A Evaristo Carriego ». In : Pugliese, Osvaldo y su orquesta : La Biandunga. Polygram 00602498863596-1-9, 1969 (CD).

Pugliese Osvaldo : «A Evaristo Carriego ». In : Pugliese Osvaldo : En el Teatro Colón de Buenos Aires. Vol. 1. EMI 56793, 1986 (33 tours).

Piazzolla, Astor / Pugliese, Osvaldo : «A Evaristo Carriego ». In : Piazzolla, Astor / Pugliese, Osvaldo : Finally together. Vol. 2. Lucho 8315392 , 1989 (CD).

Trío Eduardo Rovira : «A Evaristo Carriego ». In : Trío Eduardo Rovira : Tango En La Universidad. Edul ED 004, 1966 (33 tours).

\section{Sitographie}

www.todotango.com (consultation 01.02.2019).

https://tango.info (consultation 01.02.2019).

https://hermanotango.com (consultation 01.02.2019).

www.terapiatanguera.com.ar (consultation 01.02.2019). 